\title{
Some new generalizations for $m$-convexity via new conformable fractional integral operators
}

\author{
Ahmet Ocak Akdemir, Erhan Deniz, Ebru Yüksel
}

\begin{abstract}
In this paper, some new generalizations for $m$-convex functions have been given by using an integral identity via new conformable fractional integrals and some further properties. It is pointed out that special cases of our findings gave some earlier inequalities involving Riemann-Liouville fractional integrals.
\end{abstract}

\section{INTRODUCTION}

We will recall some definitions as follows.

Definition 1.1. A function $f: I \subseteq \mathbb{R} \rightarrow \mathbb{R}$ is said to be convex if the inequality

$$
f(t x+(1-t) y) \leq t f(x)+(1-t) f(y)
$$

holds for all $x, y \in I$ and $t \in[0,1]$.

$m$-convexity was defined by Toader as follow:

Definition 1.2. (See [6]) The function $f:[0, b] \rightarrow \mathbb{R}, b>0$, is said to be $m$-convex, where $m \in[0,1]$,we have

$$
f(t x+m(1-t) y) \leq t f(x)+m(1-t) f(y)
$$

for all $x, y \in[0, b]$ and $t \in[0,1]$.

New results, generalizations and improvements for integral inequalities via different kinds of convex functions including $m$-convexity can be found in [6]-[14].

Some fractional integral operators generalize the some other fractional integrals, in special cases, as in the following integral operator. Jarad et. al. [2] has defined a new fractional integral operator. Also, they gave some properties and relations between the some other fractional integral operators, as

2010 Mathematics Subject Classification. Primary: 26D15.

Key words and phrases. New Conformable fractional integrals, $m$-convexity, Euler Beta function.

Full paper. Received 31 July 2018, revised 5 December 2018, accepted 16 October 2019, available online 22 October 2019. 
Riemann-Liouville fractional integral, Hadamard fractional integrals, generalized fractional integral operators etc., with this operator.

Let $\beta \in \mathbb{C}, \operatorname{Re}(\beta)>0$, then the left and right sided fractional conformable integral operators has defined respectively, as follows;

$$
\begin{aligned}
& { }_{a}^{\beta} \mathfrak{J}^{\alpha} f(x)=\frac{1}{\Gamma(\beta)} \int_{a}^{x}\left(\frac{(x-a)^{\alpha}-(t-a)^{\alpha}}{\alpha}\right)^{\beta-1} \frac{f(t)}{(t-a)^{1-\alpha}} \mathrm{d} t ; \\
& { }^{\beta} \mathfrak{J}_{b}^{\alpha} f(x)=\frac{1}{\Gamma(\beta)} \int_{x}^{b}\left(\frac{(b-x)^{\alpha}-(b-t)^{\alpha}}{\alpha}\right)^{\beta-1} \frac{f(t)}{(b-t)^{1-\alpha}} \mathrm{d} t .
\end{aligned}
$$

For recent results, generalizations and improvements see the papers [1]-[4].

The main purpose of this paper is to give some new results as generalizations of the previous results for $m$-convexity via new conformable fractional integral operators.

\section{Main Results}

In order to prove our main theorems, we need the following lemma.

Lemma 2.1. (See [5]) Let $f:[a, b] \rightarrow \mathbb{R}$ be a differentiable function on $(a, b)$ with $a<b$ and $f^{\prime} \in L[a, b]$. Then the following equality holds for fractional conformable integrals:

$$
\begin{aligned}
& \frac{(x-a)^{\alpha \beta}+(b-x)^{\alpha \beta}}{(b-a) \alpha^{\beta}} f(x)-\frac{\Gamma(\beta+1)}{b-a}\left[{ }_{x}^{\beta} \mathfrak{J}^{\alpha} f(b)+{ }^{\beta} \mathfrak{J}_{x}^{\alpha} f(a)\right] \\
= & \frac{(x-a)^{\alpha \beta+1}}{b-a} \int_{0}^{1}\left(\frac{1-(1-t)^{\alpha}}{\alpha}\right)^{\beta} f^{\prime}(t x+(1-t) a) \mathrm{d} t \\
& +\frac{(b-x)^{\alpha \beta+1}}{b-a} \int_{0}^{1}\left(\frac{1-(1-t)^{\alpha}}{\alpha}\right)^{\beta} f^{\prime}(t x+(1-t) b) \mathrm{d} t
\end{aligned}
$$

where $\alpha, \beta>0$.

Theorem 2.1. Let $f:[a, b] \rightarrow \mathbb{R}$ be a differentiable function on $(a, b)$ with $a<b$ and $f^{\prime} \in L[a, b]$. If $\left|f^{\prime}\right|$ is $m$-convex on $[a, b]$ for some fixed $m \in(0,1]$, then the following inequality holds for fractional conformable integrals:

$$
\begin{aligned}
& \left|\frac{(x-a)^{\alpha \beta}+(b-x)^{\alpha \beta}}{(b-a) \alpha^{\beta}} f(x)-\frac{\Gamma(\beta+1)}{b-a}\left[{ }_{x}^{\beta} \mathfrak{J}^{\alpha} f(b)+{ }^{\beta} \mathfrak{J}_{x}^{\alpha} f(a)\right]\right| \\
\leq & \frac{m \alpha \beta}{2(\alpha \beta+2)(b-a) \alpha^{\beta}}\left(\left|f^{\prime}\left(\frac{a}{m}\right)\right|(x-a)^{\alpha \beta+1}+\left|f^{\prime}\left(\frac{b}{m}\right)\right|(b-x)^{\alpha \beta+1}\right) \\
& +\frac{\left|f^{\prime}(x)\right|}{\alpha^{\beta}(b-a)}\left(\frac{\alpha^{2} \beta^{2}+3 \alpha \beta}{2\left(\alpha^{2} \beta^{2}+3 \alpha \beta+2\right)}\right)\left((x-a)^{\alpha \beta+1}+(b-x)^{\alpha \beta+1}\right)
\end{aligned}
$$

where $\alpha>0, \beta>1$. 
Proof. Since $\left|f^{\prime}\right|$ is $m$-convex on $[a, b]$ and by Lemma 2.1, we can write

$$
\begin{aligned}
& \left|\frac{(x-a)^{\alpha \beta}+(b-x)^{\alpha \beta}}{(b-a) \alpha^{\beta}} f(x)-\frac{\Gamma(\beta+1)}{b-a}\left[{ }_{x}^{\beta} \mathfrak{J}^{\alpha} f(b)+{ }^{\beta} \mathfrak{J}_{x}^{\alpha} f(a)\right]\right| \\
\leq & \frac{(x-a)^{\alpha \beta+1}}{b-a} \int_{0}^{1}\left(\frac{1-(1-t)^{\alpha}}{\alpha}\right)^{\beta}\left|f^{\prime}(t x+(1-t) a)\right| \mathrm{d} t \\
& +\frac{(b-x)^{\alpha \beta+1}}{b-a} \int_{0}^{1}\left(\frac{1-(1-t)^{\alpha}}{\alpha}\right)^{\beta}\left|f^{\prime}(t x+(1-t) b)\right| \mathrm{d} t \\
= & \frac{(x-a)^{\alpha \beta+1}}{b-a} \int_{0}^{1}\left(\frac{1-(1-t)^{\alpha}}{\alpha}\right)^{\beta}\left|f^{\prime}\left(t x+m(1-t) \frac{a}{m}\right)\right| \mathrm{d} t \\
& +\frac{(b-x)^{\alpha \beta+1}}{b-a} \int_{0}^{1}\left(\frac{1-(1-t)^{\alpha}}{\alpha}\right)^{\beta}\left|f^{\prime}\left(t x+m(1-t) \frac{b}{m}\right)\right| \mathrm{d} t \\
\leq & \frac{(x-a)^{\alpha \beta+1}}{b-a} \int_{0}^{1}\left(\frac{1-(1-t)^{\alpha}}{\alpha}\right)^{\beta}\left(t\left|f^{\prime}(x)\right|+m(1-t)\left|f^{\prime}\left(\frac{a}{m}\right)\right|\right) \mathrm{d} t \\
& +\frac{(b-x)^{\alpha \beta+1}}{b-a} \int_{0}^{1}\left(\frac{1-(1-t)^{\alpha}}{\alpha}\right)^{\beta}\left(t\left|f^{\prime}(x)\right|+m(1-t)\left|f^{\prime}\left(\frac{b}{m}\right)\right|\right) \mathrm{d} t .
\end{aligned}
$$

By using the fact that $\left|1-(1-t)^{\alpha}\right|^{\beta} \leq 1-|1-t|^{\alpha \beta}$ for $\alpha>0, \beta>1$, we can write

$$
\begin{aligned}
& \left|\frac{(x-a)^{\alpha \beta}+(b-x)^{\alpha \beta}}{(b-a) \alpha^{\beta}} f(x)-\frac{\Gamma(\beta+1)}{b-a}\left[{ }_{x}^{\beta} \mathfrak{J}^{\alpha} f(b)+{ }^{\beta} \mathfrak{J}_{x}^{\alpha} f(a)\right]\right| \\
\leq & \frac{(x-a)^{\alpha \beta+1}}{b-a} \int_{0}^{1}\left(\frac{1-|1-t|^{\alpha \beta}}{\alpha^{\beta}}\right)\left(t\left|f^{\prime}(x)\right|+m(1-t)\left|f^{\prime}\left(\frac{a}{m}\right)\right|\right) \mathrm{d} t \\
& +\frac{(b-x)^{\alpha \beta+1}}{b-a} \int_{0}^{1}\left(\frac{1-|1-t|^{\alpha \beta}}{\alpha^{\beta}}\right)\left(t\left|f^{\prime}(x)\right|+m(1-t)\left|f^{\prime}\left(\frac{b}{m}\right)\right|\right) \mathrm{d} t
\end{aligned}
$$

By computing the above integrals, the proof is completed.

Corollary 2.1. Under the assumptions of Theorem 1,

1) If we choose $m=1$, we have the following inequality;

$$
\begin{aligned}
& \left|\frac{(x-a)^{\alpha \beta}+(b-x)^{\alpha \beta}}{(b-a) \alpha^{\beta}} f(x)-\frac{\Gamma(\beta+1)}{b-a}\left[{ }_{x}^{\beta} \mathfrak{J}^{\alpha} f(b)+{ }^{\beta} \mathfrak{J}_{x}^{\alpha} f(a)\right]\right| \\
\leq & \frac{\alpha \beta}{2(\alpha \beta+2)(b-a) \alpha^{\beta}}\left(\left|f^{\prime}(a)\right|(x-a)^{\alpha \beta+1}+\left|f^{\prime}(b)\right|(b-x)^{\alpha \beta+1}\right) \\
& +\frac{f(x)}{\alpha^{\beta}(b-a)}\left(\frac{\alpha^{2} \beta^{2}+3 \alpha \beta}{2\left(\alpha^{2} \beta^{2}+3 \alpha \beta+2\right)}\right)\left((x-a)^{\alpha \beta+1}+(b-x)^{\alpha \beta+1}\right) .
\end{aligned}
$$

2) If we choose $m=1$ and $x=\frac{a+b}{2}$, we have the following inequality;

$$
\left|\frac{2^{1-\alpha \beta}(b-a)^{\alpha \beta-1}}{\alpha^{\beta}} f\left(\frac{a+b}{2}\right)-\frac{\Gamma(\beta+1)}{b-a}\left[\frac{\beta}{\frac{a+b}{2}} \mathfrak{J}^{\alpha} f(b)+{ }^{\beta} \mathfrak{J}_{\frac{a+b}{2}}^{\alpha} f(a)\right]\right|
$$




$$
\begin{aligned}
\leq & \frac{\alpha \beta(b-a)^{\alpha \beta}}{2^{\alpha \beta+2}(\alpha \beta+2) \alpha^{\beta}}\left(\left|f^{\prime}(a)\right|+\left|f^{\prime}(b)\right|\right) \\
& +\frac{(b-a)^{\alpha \beta}}{\alpha^{\beta} 2^{\alpha \beta+1}}\left(\frac{\alpha^{2} \beta^{2}+3 \alpha \beta}{\left(\alpha^{2} \beta^{2}+3 \alpha \beta+2\right)}\right)\left|f^{\prime}\left(\frac{a+b}{2}\right)\right| .
\end{aligned}
$$

Theorem 2.2. Let $f:[a, b] \rightarrow \mathbb{R}$ be a differentiable function on $(a, b)$ with $a<b$ and $f^{\prime} \in L[a, b]$. If $\left|f^{\prime}\right|^{q}$ is $m$-convex on $[a, b]$ for some fixed $m \in(0,1]$ and $p, q>1$, then the following inequality holds for fractional conformable integrals:

$$
\begin{aligned}
& \left|\frac{(x-a)^{\alpha \beta}+(b-x)^{\alpha \beta}}{(b-a) \alpha^{\beta}} f(x)-\frac{\Gamma(\beta+1)}{b-a}\left[{ }_{x}^{\beta} \mathfrak{J}^{\alpha} f(b)+{ }^{\beta} \mathfrak{J}_{x}^{\alpha} f(a)\right]\right| \\
\leq & \frac{(x-a)^{\alpha \beta+1}}{b-a}\left(\frac{B\left(\beta p+1, \frac{1}{\alpha}\right)}{\alpha^{\beta+1}}\right)^{\frac{1}{p}}\left(\frac{\left|f^{\prime}(x)\right|^{q}+m\left|f^{\prime}\left(\frac{a}{m}\right)\right|^{q}}{2}\right)^{\frac{1}{q}} \\
& +\frac{(b-x)^{\alpha \beta+1}}{b-a}\left(\frac{B\left(\beta p+1, \frac{1}{\alpha}\right)}{\alpha^{\beta+1}}\right)^{\frac{1}{p}}\left(\frac{\left|f^{\prime}(x)\right|^{q}+m\left|f^{\prime}\left(\frac{b}{m}\right)\right|^{q}}{2}\right)^{\frac{1}{q}} .
\end{aligned}
$$

where $\frac{1}{p}+\frac{1}{q}=1, \alpha, \beta>0, B(x, y)$ is Euler Beta function.

Proof. From Lemma 2.1 and using the Hölder inequality, we have

$$
\begin{gathered}
\quad\left|\frac{(x-a)^{\alpha \beta}+(b-x)^{\alpha \beta}}{(b-a) \alpha^{\beta}} f(x)-\frac{\Gamma(\beta+1)}{b-a}\left[{ }_{x}^{\beta} \mathfrak{J}^{\alpha} f(b)+{ }^{\beta} \mathfrak{J}_{x}^{\alpha} f(a)\right]\right| \\
\leq \frac{(x-a)^{\alpha \beta+1}}{b-a}\left(\int_{0}^{1}\left(\frac{1-(1-t)^{\alpha}}{\alpha}\right)^{\beta p} \mathrm{~d} t\right)^{\frac{1}{p}} \cdot \\
\cdot\left(\int_{0}^{1}\left(t\left|f^{\prime}(x)\right|^{q}+m(1-t)\left|f^{\prime}\left(\frac{a}{m}\right)\right|^{q}\right) \mathrm{d} t\right)^{\frac{1}{q}} \\
+\frac{(b-x)^{\alpha \beta+1}}{b-a}\left(\int_{0}^{1}\left(\frac{1-(1-t)^{\alpha}}{\alpha}\right)^{\beta p} d t\right)^{\frac{1}{p}} \cdot \\
\cdots\left(\int_{0}^{1}\left(t\left|f^{\prime}(x)\right|^{q}+m(1-t)\left|f^{\prime}\left(\frac{b}{m}\right)\right|^{q}\right) \mathrm{d} t\right)^{\frac{1}{q}} .
\end{gathered}
$$

If the above integrals are calculated, we obtain

$$
\begin{aligned}
& \left|\frac{(x-a)^{\alpha \beta}+(b-x)^{\alpha \beta}}{(b-a) \alpha^{\beta}} f(x)-\frac{\Gamma(\beta+1)}{b-a}\left[{ }_{x}^{\beta} \mathfrak{J}^{\alpha} f(b)+{ }^{\beta} \mathfrak{J}_{x}^{\alpha} f(a)\right]\right| \\
\leq & \frac{(x-a)^{\alpha \beta+1}}{b-a}\left(\frac{B\left(\beta p+1, \frac{1}{\alpha}\right)}{\alpha^{\beta+1}}\right)^{\frac{1}{p}}\left(\frac{\left|f^{\prime}(x)\right|^{q}+m\left|f^{\prime}\left(\frac{a}{m}\right)\right|^{q}}{2}\right)^{\frac{1}{q}}
\end{aligned}
$$




$$
+\frac{(b-x)^{\alpha \beta+1}}{b-a}\left(\frac{B\left(\beta p+1, \frac{1}{\alpha}\right)}{\alpha^{\beta+1}}\right)^{\frac{1}{p}}\left(\frac{\left|f^{\prime}(x)\right|^{q}+m\left|f^{\prime}\left(\frac{b}{m}\right)\right|^{q}}{2}\right)^{\frac{1}{q}} .
$$

The proof is completed.

Corollary 2.2. Under the assumptions of Theorem 2,

1) If we choose $m=1$, we have the following inequality;

$$
\begin{aligned}
& \left|\frac{(x-a)^{\alpha \beta}+(b-x)^{\alpha \beta}}{(b-a) \alpha^{\beta}} f(x)-\frac{\Gamma(\beta+1)}{b-a}\left[{ }_{x}^{\beta} \mathfrak{J}^{\alpha} f(b)+{ }^{\beta} \mathfrak{J}_{x}^{\alpha} f(a)\right]\right| \\
\leq & \frac{(x-a)^{\alpha \beta+1}}{b-a}\left(\frac{B\left(\beta p+1, \frac{1}{\alpha}\right)}{\alpha^{\beta+1}}\right)^{\frac{1}{p}}\left(\frac{\left|f^{\prime}(x)\right|^{q}+\left|f^{\prime}(a)\right|^{q}}{2}\right)^{\frac{1}{q}} \\
& +\frac{(b-x)^{\alpha \beta+1}}{b-a}\left(\frac{B\left(\beta p+1, \frac{1}{\alpha}\right)}{\alpha^{\beta+1}}\right)^{\frac{1}{p}}\left(\frac{\left|f^{\prime}(x)\right|^{q}+\left|f^{\prime}(b)\right|^{q}}{2}\right)^{\frac{1}{q}} .
\end{aligned}
$$

2) If we choose $m=1$ and $x=\frac{a+b}{2}$, we have the following inequality;

$$
\begin{aligned}
& \left|\frac{2^{1-\alpha \beta}(b-a)^{\alpha \beta-1}}{\alpha^{\beta}} f\left(\frac{a+b}{2}\right)-\frac{\Gamma(\beta+1)}{b-a}\left[{ }_{\frac{a+b}{2}}^{\beta} \mathfrak{J}^{\alpha} f(b)+{ }^{\beta} \mathfrak{J}_{\frac{a+b}{2}}^{\alpha} f(a)\right]\right| \\
\leq & \frac{(b-a)^{\alpha \beta}}{2^{\alpha \beta+1}}\left(\frac{B\left(\beta p+1, \frac{1}{\alpha}\right)}{\alpha^{\beta+1}}\right)^{\frac{1}{p}}\left(\frac{\left|f^{\prime}\left(\frac{a+b}{2}\right)\right|^{q}+\left|f^{\prime}(a)\right|^{q}}{2}\right)^{\frac{1}{q}} \\
& +\frac{(b-a)^{\alpha \beta}}{2^{\alpha \beta+1}}\left(\frac{B\left(\beta p+1, \frac{1}{\alpha}\right)}{\alpha^{\beta+1}}\right)^{\frac{1}{p}}\left(\frac{\left|f^{\prime}\left(\frac{a+b}{2}\right)\right|^{q}+\left|f^{\prime}(b)\right|^{q}}{2}\right)^{\frac{1}{q}} .
\end{aligned}
$$

Remark 2.1. If we choose $\left|f^{\prime}\right|<M$ in Corollary 2 (i), we obtain Theorem 2.2 of [5].

Theorem 2.3. Let $f:[a, b] \rightarrow \mathbb{R}$ be a differentiable function on $(a, b)$ with $a<b$ and $f^{\prime} \in L[a, b]$. If $\left|f^{\prime}\right|^{q}$ is $m$-convex on $[a, b]$ for some fixed $m \in(0,1]$ and $q \geq 1$, then the following inequality holds for fractional conformable integrals:

$$
\begin{aligned}
& \left|\frac{(x-a)^{\alpha \beta}+(b-x)^{\alpha \beta}}{(b-a) \alpha^{\beta}} f(x)-\frac{\Gamma(\beta+1)}{b-a}\left[\begin{array}{l}
\beta \\
x \\
\mathfrak{J}^{\alpha}
\end{array} f(b)+{ }^{\beta} \mathfrak{J}_{x}^{\alpha} f(a)\right]\right| \\
\leq & \frac{(x-a)^{\alpha \beta+1}}{b-a}\left(\frac{B\left(\frac{1}{\alpha}, \beta+1\right)}{\alpha^{\beta+1}}\right)^{1-\frac{1}{q}} \\
& \times\left(\frac{\left|f^{\prime}(x)\right|^{q}}{\alpha^{\beta}}\left(\frac{1}{2}-B(2, \alpha \beta+1)\right)+\frac{m\left|f^{\prime}\left(\frac{a}{m}\right)\right|^{q}}{\alpha^{\beta}}\left(\frac{\alpha \beta}{2(\alpha \beta+2)}\right)\right)^{\frac{1}{q}} \\
& +\frac{(b-x)^{\alpha \beta+1}}{b-a}\left(\frac{B\left(\frac{1}{\alpha}, \beta+1\right)}{\alpha^{\beta+1}}\right)^{1-\frac{1}{q}}
\end{aligned}
$$




$$
\times\left(\frac{\left|f^{\prime}(x)\right|^{q}}{\alpha^{\beta}}\left(\frac{1}{2}-B(2, \alpha \beta+1)\right)+\frac{m\left|f^{\prime}\left(\frac{b}{m}\right)\right|^{q}}{\alpha^{\beta}}\left(\frac{\alpha \beta}{2(\alpha \beta+2)}\right)\right)^{\frac{1}{q}} .
$$

where $\alpha>0, \beta>1, B(x, y)$ is Euler Beta function.

Proof. Since $\left|f^{\prime}\right|^{q}$ is $m$-convex on $[a, b]$ for $q \geq 1$, from Lemma 2.1 and the well known power mean integral inequality, we can write

$$
\begin{aligned}
& \left|\frac{(x-a)^{\alpha \beta}+(b-x)^{\alpha \beta}}{(b-a) \alpha^{\beta}} f(x)-\frac{\Gamma(\beta+1)}{b-a}\left[{ }_{x}^{\beta} \mathfrak{J}^{\alpha} f(b)+{ }^{\beta} \mathfrak{J}_{x}^{\alpha} f(a)\right]\right| \\
\leq & \frac{(x-a)^{\alpha \beta+1}}{b-a}\left(\int_{0}^{1}\left(\frac{1-(1-t)^{\alpha}}{\alpha}\right)^{\beta} \mathrm{d} t\right)^{1-\frac{1}{q}} \\
& \times\left(\int_{0}^{1}\left(\frac{1-(1-t)^{\alpha}}{\alpha}\right)^{\beta}\left(t\left|f^{\prime}(x)\right|^{q}+m(1-t)\left|f^{\prime}\left(\frac{a}{m}\right)\right|^{q}\right) \mathrm{d} t\right)^{\frac{1}{q}} \\
& +\frac{(b-x)^{\alpha \beta+1}}{b-a}\left(\int_{0}^{1}\left(\frac{1-(1-t)^{\alpha}}{\alpha}\right)^{\beta} \mathrm{d} t\right)^{1-\frac{1}{q}} \\
& \times\left(\int_{0}^{1}\left(\frac{1-(1-t)^{\alpha}}{\alpha}\right)^{\beta}\left(t\left|f^{\prime}(x)\right|^{q}+m(1-t)\left|f^{\prime}\left(\frac{b}{m}\right)\right|^{q}\right) \mathrm{d} t\right)^{\frac{1}{q}} .
\end{aligned}
$$

By a simple computation and by using the fact that $\left|1-(1-t)^{\alpha}\right|^{\beta} \leq 1-$ $|1-t|^{\alpha \beta}$ for $\alpha>0, \beta>1$, we get

$$
\begin{aligned}
& \left|\frac{(x-a)^{\alpha \beta}+(b-x)^{\alpha \beta}}{(b-a) \alpha^{\beta}} f(x)-\frac{\Gamma(\beta+1)}{b-a}\left[{ }_{x}^{\beta} \mathfrak{J}^{\alpha} f(b)+{ }^{\beta} \mathfrak{J}_{x}^{\alpha} f(a)\right]\right| \\
\leq & \frac{(x-a)^{\alpha \beta+1}}{b-a}\left(\frac{B\left(\frac{1}{\alpha}, \beta+1\right)}{\alpha^{\beta+1}}\right)^{1-\frac{1}{q}} \\
& \times\left(\frac{\left|f^{\prime}(x)\right|^{q}}{\alpha^{\beta}}\left(\frac{1}{2}-B(2, \alpha \beta+1)\right)+\frac{m\left|f^{\prime}\left(\frac{a}{m}\right)\right|^{q}}{\alpha^{\beta}}\left(\frac{\alpha \beta}{2(\alpha \beta+2)}\right)\right)^{\frac{1}{q}} \\
& +\frac{(b-x)^{\alpha \beta+1}}{b-a}\left(\frac{B\left(\frac{1}{\alpha}, \beta+1\right)}{\alpha^{\beta+1}}\right)^{1-\frac{1}{q}} \\
& \times\left(\frac{\left|f^{\prime}(x)\right|^{q}}{\alpha^{\beta}}\left(\frac{1}{2}-B(2, \alpha \beta+1)\right)+\frac{m\left|f^{\prime}\left(\frac{b}{m}\right)\right|^{q}}{\alpha^{\beta}}\left(\frac{\alpha \beta}{2(\alpha \beta+2)}\right)\right)^{\frac{1}{q}} .
\end{aligned}
$$

the result is obtained and the proof is completed. 
Corollary 2.3. Under the assumptions of Theorem 3:

1) If we choose $m=1$, we have the following inequality:

$$
\begin{aligned}
& \quad\left|\frac{(x-a)^{\alpha \beta}+(b-x)^{\alpha \beta}}{(b-a) \alpha^{\beta}} f(x)-\frac{\Gamma(\beta+1)}{b-a}\left[{ }_{x}^{\beta} \mathfrak{J}^{\alpha} f(b)+{ }^{\beta} \mathfrak{J}_{x}^{\alpha} f(a)\right]\right| \\
& \leq \quad \frac{(x-a)^{\alpha \beta+1}}{b-a}\left(\frac{B\left(\frac{1}{\alpha}, \beta+1\right)}{\alpha^{\beta+1}}\right)^{1-\frac{1}{q}} \\
& \quad \times\left(\frac{\left|f^{\prime}(x)\right|^{q}}{\alpha^{\beta}}\left(\frac{1}{2}-B(2, \alpha \beta+1)\right)+\frac{\left|f^{\prime}(a)\right|^{q}}{\alpha^{\beta}}\left(\frac{\alpha \beta}{2(\alpha \beta+2)}\right)\right)^{\frac{1}{q}} \\
& +\frac{(b-x)^{\alpha \beta+1}}{b-a}\left(\frac{B\left(\frac{1}{\alpha}, \beta+1\right)}{\alpha^{\beta+1}}\right)^{1-\frac{1}{q}} \\
& \quad \times\left(\frac{\left|f^{\prime}(x)\right|^{q}}{\alpha^{\beta}}\left(\frac{1}{2}-B(2, \alpha \beta+1)\right)+\frac{\left|f^{\prime}(b)\right|^{q}}{\alpha^{\beta}}\left(\frac{\alpha \beta}{2(\alpha \beta+2)}\right)\right)^{\frac{1}{q}} .
\end{aligned}
$$

2) If we choose $m=1$ and $x=\frac{a+b}{2}$, we have the following inequality:

$$
\begin{aligned}
& \left|\frac{2^{1-\alpha \beta}(b-a)^{\alpha \beta-1}}{\alpha^{\beta}} f\left(\frac{a+b}{2}\right)-\frac{\Gamma(\beta+1)}{b-a}\left[{ }_{\frac{a+b}{2}}^{\beta} \mathfrak{J}^{\alpha} f(b)+{ }^{\beta} \mathfrak{J}_{\frac{a+b}{2}}^{\alpha} f(a)\right]\right| \\
\leq & \frac{(b-a)^{\alpha \beta}}{2^{\alpha \beta+1}}\left(\frac{B\left(\frac{1}{\alpha}, \beta+1\right)}{\alpha^{\beta+1}}\right){ }^{1-\frac{1}{q}} \\
& \times\left(\frac{\left|f^{\prime}\left(\frac{a+b}{2}\right)\right|^{q}}{\alpha^{\beta}}\left(\frac{1}{2}-B(2, \alpha \beta+1)\right)+\frac{\left|f^{\prime}(a)\right|^{q}}{\alpha^{\beta}}\left(\frac{\alpha \beta}{2(\alpha \beta+2)}\right)\right)^{\frac{1}{q}} \\
& +\frac{(b-a)^{\alpha \beta}}{2^{\alpha \beta+1}}\left(\frac{B\left(\frac{1}{\alpha}, \beta+1\right)}{\alpha^{\beta+1}}\right)^{1-\frac{1}{q}} \\
& \times\left(\frac{\left|f^{\prime}\left(\frac{a+b}{2}\right)\right|^{q}}{\alpha^{\beta}}\left(\frac{1}{2}-B(2, \alpha \beta+1)\right)+\frac{\left|f^{\prime}(b)\right|^{q}}{\alpha^{\beta}}\left(\frac{\alpha \beta}{2(\alpha \beta+2)}\right)\right)^{\frac{1}{q}} .
\end{aligned}
$$

Remark 2.2. If we choose $\left|f^{\prime}\right|<M$ in Corollary 3 (i), we obtain Theorem 2.3 of [5]. 


\section{REFERENCES}

[1] A. Gözpınar, Some Hermite-Hadamard Type Inequalities For Convex Functions Via New Fractional Conformable Integrals And Related Inequalities, American Institute of Physics Conference Proceedings, 1991 (2018), 020006.

[2] F. Jarad, E. Uğurlu, T. Abdeljawad and D. Baleanu, On a new class of fractional operators, Advances in Difference Equations, 2017 (2017), Article ID: 247, 16 pages.

[3] E. Set, A. Gözpınar, F. Demirci, Hermite-Hadamard type inequalities for quasi-convex functions via new fractional conformable integrals, American Institute of Physics Conference Proceedings, 1991 (2018), 020002.

[4] E. Set, A. Karaoğlan, A. Gözpınar, Some inequalities related to different convex functions via new fractional conformable integrals, American Institute of Physics Conference Proceedings, 1991 (2018), 020012.

[5] E. Set, A. O. Akdemir, A. Gözpınar and F. Jarad, Ostrowski type inequalities via new fractional comformable integrals, AIMS Mathematics, 4 (6) (2019), 1684-1697.

[6] G. Toader, Some generalizations of the convexity, Proceedings of The Colloquium On Approximation and Optimization, Univ. Cluj-Napoca, Cluj-Napoca, (1984), 329-338

[7] M. K. Bakula, M. E. Özdemir and J. Pečarić, Hadamard type inequalities for $m$-convex and $(\alpha, m)$-convex functions, Journal of Inequalities in Pure and Applied Mathematics, (9) (4) (2008), Article 96.

[8] S. S. Dragomir, I. Gomm, Hermite-Hadamard type inequalities for $(m, M)-\Psi-$ convex functions when $\Psi=-\ln$, Mathematica Moravica, 22 (1) (2018), 65-79.

[9] M. K. Bakula, J. Pečarić and M. Ribičić, Companion inequalities to Jensen's inequality for $m$-convex and $(\alpha, m)$-convex functions, Journal of Inequalities in Pure and Applied Mathematics, (7) (5) (2006), Article 194.

[10] A. Akkurt, H. Yıldırım, On Hermite-Hadamard-Fejér type inequalities for convex functions via fractional integrals, Mathematica Moravica, 21 (1) (2017), 105-123.

[11] M.E. Özdemir, A. Ekinci and A.O. Akdemir, Generalizations of integral inequalities for functions whose second derivatives are convex and $m$-convex, Miskolc Mathematical Notes, 13-2 (2012), 441-457.

[12] M.E. Özdemir, A.O. Akdemir and A. Ekinci, New Hadamard-type inequalities for functions whose derivatives are $(\alpha, m)$-convex functions, Tbilisi Mathematical Journal, 7 (2) (2014), 61-72.

[13] M.E. Özdemir and A. Ekinci, Generalized integral inequalities for convex functions, Mathematical Inequalities and Applications, 19 (4) (2016), 1429-1439.

[14] S. S. Dragomir and G. Toader, Some inequalities for $m$-convex functions, Studia Universitatis Babeş-Bolyai Mathematica, 38 (1) (1993), 21-28. 
Ahmet Ocak Akdemír

AĞri İbrahim Çeçen University

Faculty of Science and Letters

Department of Mathematics

04100, AĞRI

TURKEY

E-mail address: aocakakdemir@gmail.com

\section{Erhan Deniz}

KAFKAS UNIVERSITY

Faculty of Science and Letters

Department of Mathematics

36100 KARS

TURKEY

E-mail address: edeniz36@gmail.com

\section{EBRU YÜKSEL}

AĞri İbrahim Çeçen University

Faculty of Science and Letters

Department of Mathematics

04100 AĞRI

TURKEY

E-mail address: eyuksel@agri.edu.tr 